\title{
The down syndrome biomarker initiative (DSBI) pilot: proof of concept for deep phenotyping of Alzheimer's disease biomarkers in down syndrome
}

\author{
Michael S. Rafii ${ }^{1 *}$, Hannah Wishnek ${ }^{1}$, James B. Brewer ${ }^{1}$, Michael C. Donohue ${ }^{2}$, \\ Seth Ness ${ }^{3}$, William C. Mobley ${ }^{1}$, Paul S. Aisen ${ }^{2}$ and Robert A. Rissman ${ }^{1}$

\begin{abstract}
Alzheimer's Disease Cooperative Study, Department of Neurosciences, UC San Diego School of Medicine, University of California, San Diego, La Jolla, CA, USA, ${ }^{2}$ Department of Neurology, University of Southern California, Los Angeles, CA, USA, ${ }^{3}$ Janssen Research and Development LLC, Titusville, NJ, USA
\end{abstract}

\section{OPEN ACCESS}

Edited by:

Roger H. Reeves,

Johns Hopkins University, USA

Reviewed by: Shane M. O'Mara, Trinity College Dublin, Ireland Benjamni Handen, University of Pittsburgh, USA

*Correspondence: Michael S. Rafii,

Alzheimer's Disease

Cooperative Study,

Department of Neurosciences, UC San Diego School of Medicine, University of California, San Diego, 9500 Gilman Drive, MC 0949,

La Jolla, CA 92094-0949, USA mrafii@ucsd.edu

Received: 29 April 2015 Accepted: 21 August 2015 Published: 14 September 2015

Citation:

Rafii MS, Wishnek H, Brewer JB, Donohue MC, Ness S, Mobley WC, Aisen PS and Rissman RA (2015)

The down syndrome biomarker initiative (DSBI) pilot: proof of concept for deep phenotyping of Alzheimer's disease biomarkers in down syndrome.

Front. Behav. Neurosci. 9:239. doi: 10.3389/fnbeh.2015.00239
To gain further knowledge on the preclinical phase of Alzheimer's disease (AD), we sought to characterize cognitive performance, neuroimaging and plasma-based $A D$ biomarkers in a cohort of non-demented adults with down syndrome (DS). The goal of the down syndrome biomarker Initiative (DSBI) pilot is to test feasibility of this approach for future multicenter studies. We enrolled 12 non-demented participants with DS between the ages of 30-60 years old. Participants underwent extensive cognitive testing, volumetric MRI, amyloid positron emission tomography (PET; 18F-florbetapir), fluorodeoxyglucose (FDG) PET (18F-fluorodeoxyglucose) and retinal amyloid imaging. In addition, plasma beta-amyloid (A $\beta$ ) species were measured and Apolipoprotein $E$ (ApoE) genotyping was performed. Results from our multimodal analysis suggest greater hippocampal atrophy with amyloid load. Additionally, we identified an inverse relationship between amyloid load and regional glucose metabolism. Cognitive and functional measures did not correlate with amyloid load in DS but did correlate with regional FDG PET measures. Biomarkers of AD can be readily studied in adults with DS as in other preclinical AD populations. Importantly, all subjects in this feasibility study were able to complete all test procedures. The data indicate that a large, multicenter longitudinal study is feasible to better understand the trajectories of AD biomarkers in this enriched population. This trial is registered with ClinicalTrials.gov, number NCT02141971.

Keywords: down syndrome, Alzheimer's disease, biomarkers, amyloid, MRI, PET, plasma, retinal

\section{Introduction}

The preclinical/asymptomatic stage of Alzheimer's disease (AD) has become a target for therapeutic intervention, requiring enriched populations to be more intensively studied. Individuals with Down Syndrome (DS) comprise the largest group with genetically determined AD, with a worldwide population of about six million people. In March 2013, the Alzheimer's Disease Cooperative Study (ADCS) launched a pilot study named the Down Syndrome Biomarker Initiative (DSBI; Ness et al., 2012). With the DSBI pilot, the ADCS' goal was to initiate a longitudinal biomarker 
study similar to the Alzheimer's Disease Neuroimaging Initiative (ADNI) in individuals with DS, who represent a population highly enriched for developing AD. The ultimate aim of this work is to aid the development of preventive therapies for the dementia associated with both $\mathrm{DS}$ and $\mathrm{AD}$, based on the apparent common pathogenic role of beta amyloid $(A \beta)$ in the two conditions.

The tight link between genetic determinants of $\mathrm{AD}$ and the overproduction of $A \beta$ provides compelling support for the amyloid cascade hypothesis and has been the focal point in the development of disease-modifying drugs for AD (for review, Sperling et al., 2011). We hypothesize that disease-modifying treatments for $\mathrm{AD}$ and $\mathrm{DS}$ should begin prior to the onset of cognitive symptoms to prevent extensive neurodegeneration and thus necessitate a clear understanding of biomarker changes throughout the course of the disease.

The study of DS provides a unique opportunity to characterize the preclinical changes associated with predisposition to $\mathrm{AD}$. DS, or trisomy 21, affects 400,000 people in the U.S. with an incidence of $1 / 691$ live births (Parker et al., 2010) and is caused by meiotic non-disjunction, leading to an extra copy of chromosome 21, on which the APP gene resides.

Recent data suggest that AD biomarker changes in DS are similar to those observed in familial and sporadic AD. For example, studies demonstrate a six-fold increase in plasma $\mathrm{A} \beta$ in individuals with DS as compared to age-matched nonDS individuals (Schupf et al., 2001, 2007, 2010) and $A \beta$ positron emission tomography (PET) imaging data in DS are consistent with AD patients (Sabbagh et al., 2011, 2015; Handen et al., 2012). Furthermore, as seen in familial and sporadic $\mathrm{AD}$, presence of the Apolipoprotein $\mathrm{E}$ (ApoE) $\varepsilon 4$ allele is generally associated with greater accumulation of $A \beta$ plaques in the brains of adults with DS (Hyman et al., 1995; Lemere et al., 1996). Presence of ApoE $\varepsilon 4$ allele is also associated with an earlier age of onset of dementia (Schupf et al., 1996; Deb et al., 2000; Coppus et al., 2008; Prasher et al., 2008).

Postmortem studies indicate that adults with DS have a similar, prominent pattern of cerebral atrophy involving the medial temporal lobe structures, as seen in the early stages of AD (Hof et al., 1995; Teipel et al., 2004; Mullins et al., 2013). Volumetric magnetic resonance imaging (MRI) studies of age-related brain changes in DS demonstrate the same pattern of hippocampal-specific atrophy observed in AD. Furthermore, the hippocampal atrophy in DS correlates with changes in memory measures (Krasuski et al., 2002; Beacher et al., 2010). Hypometabolism on regional fluorodeoxyglucose (FDG) PET also correlates with onset of dementia in older adults with DS (Schapiro et al., 1992a,b; Pietrini et al., 1997).

In this study, we collected structural MRI, A $\beta$ PET, FDG PET, retinal $A \beta$, plasma $A \beta$ species, and cognitive performance measurements in a cohort of 12 non-demented adults with DS aged 30-60. Our goal was to establish feasibility of conducting a biomarker-intensive study in adults with DS.

\section{Materials and Methods}

\section{Study Design and Participants}

The DSBI pilot enrolled 12 non-demented subjects for a 3year longitudinal study of AD biomarkers (see Table 1 for Schedule of Events). The present analysis is restricted to the baseline data. Four non-demented subjects were in each age range: $30-40,40-50$ and 50-60. Inclusion criteria limited enrollment to individuals having a chromosome karyotype of DS due to Trisomy 21. Subjects were required to have a caregiver, absence of other neurological and psychiatric disorders, and be capable of and willing to perform study procedures. Having a clinical diagnosis of dementia was considered exclusionary as was presence of 6 months of progressive cognitive or functional decline as per ICD-10 criteria (Sheehan et al., 2015). Exclusion of a diagnosis of dementia was also based on absence of evidence of recent deterioration in cognitive function found not secondary to medical illness (e.g., hypothyroidism, sleep apnea) in conjunction with absence of a significant decline in function over a period of 6 months or more. The diagnosing neurologist was experienced with dementia in DS and incorporated diagnostic recommendations from the National Task Group on Intellectual Disabilities and Dementia Practices (Moran et al., 2013). All participants or their legal representatives provided written informed consent before partaking in the study in accordance with the regulations and approval of the ethics committee

TABLE 1 | Schedule of events for DSBI pilot.

\begin{tabular}{lllcc}
\hline Visit & $\begin{array}{l}\text { Screen/ } \\
\text { BL }\end{array}$ & YR1 & YR2 & $\begin{array}{r}\text { YR3 } \\
\text { (Comp) }\end{array}$ \\
\hline Month & 0 & 12 & 24 & 36
\end{tabular}

Study Procedures

Screening/administrative

Informed consent [/assent]

Inclusion/exclusion criteria

Medical history and demographics $x$

Safety assessments

Physical examination

Vital signs

Neurocog assessments

Scales, questionnaires, etc.

Clinical laboratory assessments Hematology, Chemistry

Urinalysis

Pharmacogenomics (DNA)

ApoE

Biomarkers (eg, plasma, serum sample collection)

Plasma, serum collection

Imaging

Tau PET

Amyvid PET

FDG PET

VMRI

Retinal amyloid imaging

Ongoing subject review

Concomitant therapy

Adverse events




at the University of California, San Diego, La Jolla, CA, USA.

\section{Procedures}

Between March 2013 and January 2014, we collected data from participants including plasma samples, neuropsychological evaluations, neurological examination, ApoE genotyping, volumetric MRI, amyloid PET, FDG PET, retinal A $\beta$ imaging, and clinical assessment. Subjects came for five visits over a 5-week period for assessments to be made. Events occured in the following order: visit 1: neuropsychological and clinical assessment, neurological examination; visit 2: Amyloid PET; visit 3: MRI and blood draw; visit 4: FDG PET, visit 5: retinal $\mathrm{A} \beta$ imaging.

\section{Cognitive, Behavioral, and Functional Assessments}

Cambridge Neuropsychological Test Automated Battery (CANTAB). The CANTAB was used to assess cognition. The CANTAB is a computerized touch-screen assessment of neuropsychological function composed of a number of tests (Luciana, 2003; Smith et al., 2013). The tests selected from this battery for this study were as follows: motor control (MOT): the subject is asked upon appearance of a crossmark on the screen, to touch it as quickly and accurately as possible using the index finger of their dominant hand. This is essentially a practice routine to become skilled with regards to touchscreen use. The outcome parameter is median reaction time (RT): the subject is asked to hold the index finger on the holding button on the button box and keep it pressed until a circle on the screen lights up and then touch that circle with the index finger as quickly and accurately as possible. In the Simple condition, there is only one possible circle that will light up (Simple RT). In the five-choice condition, any of five circles can light up (five-Choice RT). Paired associated learning (PAL): the subject is shown 2-8 (max) distinct visual patterns, each at one of eight positions inside of an octagon on the screen. The task is to memorize which pattern occurred where. After the memorization stage, each pattern is shown in the center of the screen and the subject has then to touch one of eight possible positions where the pattern first occurred.

Repeatable Battery for the Assessment of Neuropsychological Status (RBANS) was developed for the dual purposes of identifying and characterizing abnormal cognitive decline in the older adult and as a neuropsychological screening battery for younger patients (Randolph et al., 1998). It is a brief, individually administered test that can be used to measure cognitive decline or improvement. The full battery is composed of 12 subtests assessing the: immediate memory, visuospatial abilities, language, attention and delayed memory. In this study, seven subtests of the RBANS were used to assess immediate and delayed memory, as well as the language capacities (subtests: list learning, story memory, list recognition, list recall, picture naming, semantic fluency, digit span).
Vineland-II Adaptive Behavior Scale (VABS-II) parent/caregiver interview form. The VABS-II measures personal and social skills such as communication, daily leaving skills, and socialization and will provide a composite score reflecting an individual's overall function. In addition, the optional maladaptive behavior index could be used. The survey interview form was administered to parents or caregivers using a semi-structured interview format (Sparrow and Havis, 2005).

Observer Memory Questionnaire-Parent Form (OMQ-PF). The OMQ-PF is a 27-item questionnaire designed to ascertain parents' perceptions of the subject's memory function. This questionnaire is comprised of items inquiring about memory function in everyday scenarios (Gonzalez et al., 2008).

Anxiety Depression and Mood Scale (ADAMS). The ADAMS is a well validated, 28 item behavior-based informant instrument designed to be used specifically with individuals with developmental disabilities to assess anxiety, depression and mood disorders (Esbensen et al., 2003). Points given for each behavior the caregiver endorses. Subscales (5) include: Manic/Hyperactive, Depressed Mood, Social Avoidance, General Anxiety, Compulsive Behavior. The ADAMS possesses a satisfactorily high alpha, with a mean alpha of 0.80 in each of the 28 items. The mean item test-retest correlation is 0.789 .

Cambridge Examination for Mental Disorders of Older People with Down's Syndrome and Others with Intellectual Disabilities (CAMDEX-DS). Cognitive status was measured using the Cambridge Cognitive Examination (CAMCOG), the cognitive section of CAMDEX, a composite index of episodic memory, orientation, language, attention, praxis and executive function previously validated for use in DS (Hon et al., 1999). The CAMCOG is appropriate for assessing cognitive function in people with intellectual disability, unlike more standard tests of cognitive function such as the Wechsler Adult Intelligence Scales. The CAMCOG incorporates, and is highly correlated with, the Mini Mental State Examination (MMSE; Blessed et al., 1991).

Dalton Dyspraxia scale for Adults with DS: evaluates simple sequences of voluntary movements expected to deteriorate with the onset and progression of dementia in $\mathrm{AD}$ among persons at all levels of premorbid intellectual disability. Participants are given points for each task they are able to perform (Dalton, 1992).

The Goodenough-Harris Draw-A-Person Test: brief paper and pencil mental age test. This assessment system analyses 14 different aspects of a drawing done by the subject (such as specific body parts and clothing) for various criteria, including presence or absence, detail, and proportion. In all, there are 64 scoring items. A standard score is recorded for the drawing, and a mental age is assigned based on this score (Goodenough and Harris, 1950).

\section{Biofluid Collection}

Blood, (separated into plasma and serum), was collected to accommodate the assay of the broadest range of the best antecedent biomarkers/analytes. Blood samples were drawn in 
two lavender-capped EDTA tubes and one red-capped BD tube. One lavender-capped tube was centrifuged at $3000 \mathrm{rpm}$ for $10 \mathrm{~min}$ to separate plasma for storage. Ten milliliter of the plasma sample was aliquoted into barcoded polypropylene vial and frozen at -80 . The second blood tube was used for serum extraction, which will be processed by allowing the samples to clot at room temperature, spun as above for plasma preparation, aliquoted and stored in barcoded polypropylene tubes at -80 . The third blood tube was used for DNA isolation using Qiamp DNA blood maxi kit (Qiagen). All biosamples were processed and stored at the ADCS Biomarker Core using standard operating procedure.

\section{Plasma A $\beta$ Analysis and Internal Standard}

Banked plasma was assayed, quantified, and quality controlled by the ADCS Biomarker Core using the MesoScale Validated $A \beta$ triplex $(A \beta 38,40,42)$ according to the manufacturer instructions. Each assay plate also included an internal standard which provided a means for adjusting plate-to-plate variation and assessing freezer storage effects, as previously described (Donohue et al., 2014). To mitigate plate-to-plate variability, plates were purchased in bulk and run consecutively.

\section{Real Time PCR for Apolipoprotein E (ApoE) Genotyping}

Genotyping for ApoE alleles was performed using real time PCR Restriction Fragment Length Polymorphism analysis by the ADCS Biomarker Core according to standard operating procedures. ApoE genotyping was performed using Applied Biosystems TaqMan SNP Genotyping Assay (C_3084793_20 and C_904973_10 corresponding to ApoE SNPs rs429358 and rs7412, respectively). The assay was run on a Bio-Rad CFX96 Touch Real Time PCR Detection System, using a cycling program of $98 \mathrm{C}$ for $2 \mathrm{~min}$. and 39 cycles of $98 \mathrm{C}$ for $15 \mathrm{~s}$ and $62 \mathrm{C}$ for $45 \mathrm{~s}$ five positive controls for each genotype and one negative control were included in each plate to ensure accurate determination.

\section{Neuroimaging \\ Volumetric MRI}

The MRI protocol included series to assess for structural pathology (T2-weighted fluid attenuated inversion recovery, T2*-weighted gradient recalled echo, and diffusion weighted imaging) along with a series modeled on the non-accelerated T1-weighted sequence from ADNI for volumetric processing (3D inversion recovery prepared spoiled gradient recalled imaging; inversion time 500, flip angle $10,1.25 \mathrm{~mm} \times 1.25 \mathrm{~mm}$ in-plane resolution, 156 sagittal slices with $1.2 \mathrm{~mm}$ spacing). Scanning was performed on a 1.5 Tesla GE Signa HDxt scanner, and radiologist overread was performed on all scans to identify any clinically significant incidental findings. NeuroQuant image preprocessing and automated segmentation was used to measure brain structure volumes (Brewer et al., 2009; Kovacevic et al., 2009; Heister et al., 2011). Briefly, this includes corrections for gradient non-linearities (Jovicich et al., 2006) and intensity non-uniformity (Sled et al., 1998) and application of probabilistic-atlas-based segmentation to automate measurement of multiple brain regions (Fischl et al., 2002). The procedure is cleared by the U.S. Food and Drug Administration and the European Medicines Agency for use in automating the identifying, labeling, and quantifying the volume of segmental brain structures identified on MR images (21 CFR 892.2050). To minimize multiple comparisons, for analysis, a single measure of medial temporal atrophy that comprises hippocampal volume loss and temporal horn ex-vacuo dilatation, "Hippocampal occupancy (HOC)," was calculated as described previously (Heister et al., 2011). This measure is simply $\mathrm{H} /(\mathrm{H}+\mathrm{T})$, where $\mathrm{H}$ is hippocampal volume and $\mathrm{T}$ is temporal horn volume.

\section{FDG PET}

FDG PET procedures were based on those used in ADNI. ${ }^{1}$ Subjects were asked to fast for at least $6 \mathrm{~h}$ prior to the scanning session. Subjects' blood glucose was checked prior to scanning and was required to be $<180 \mathrm{mg} / \mathrm{dL}$. After the injection of $5 \mathrm{mCi}$ of $18 \mathrm{~F}-\mathrm{FDG}$, subjects were kept in a quiet, dimly lit room with eyes and ears unoccluded for 30 min, after which they were placed in the Siemens EXACT HR+ 961 PET tomograph (CTI, Knoxville, TN, USA), which yielded 63 transverse sections spaced $2.43 \mathrm{~mm}$ apart with a $15.5 \mathrm{~cm}$ field of view (FOV) in $3 \mathrm{D}$ mode and $5 \mathrm{~mm}$ inplane spatial resolution full width at half maximum (FWHM). Images were acquired at an angle parallel to the canthomeatal plane and reconstructed using a ramp filter (cut-off frequency $=0.5$ cycles $/$ pixel) into $128 \times 128$ pixel images . Each subject was placed in a headholder during scanning to allow accurate positioning using a low-power neon laser. Data were acquired as $6 \times 5 \mathrm{~min}$ frames, followed by a positron transmission scan. Frames were averaged and all images were coregistered to the individual's native space MRI. For signal normalization, the brainstem was used as a reference region.

\section{Florbetapir F 18 PET}

Subjects received IV injections of $10 \mathrm{mCi}$ of Florbetapir F 18 and after $40 \mathrm{~min}$ of uptake, $10 \mathrm{~min}$ of emission data were collected by the Siemens EXACT HR+ 961 PET tomograph (CTI, Knoxville, TN, USA), which yielded 63 transverse sections spaced $2.43,3.5 \mathrm{~mm}$ apart with a $15.5 \mathrm{~cm}$ FOV in $3 \mathrm{D}$ mode, with $4 \mathrm{~mm}$ in-plane spatial resolution (FWHM). Images were acquired at an angle parallel to the cantho-meatal plane and reconstructed using a Hann filter (cut-off frequency $=0.5$ cycles/pixel) into $128 \times 128$ pixel images. Each subject was placed in a headholder during scanning to allow accurate positioning using a low-power neon laser. All PET scans were supervised. Statistical analysis was performed as for FDG, except, for florbetapir, the cerebellum was used as the reference region for signal normalization.

\footnotetext{
${ }^{1}$ http://www.adni-info.org/Scientists/doc/ADNI2_PET\%20Tech_ManualVersion_4_2014Oct27_CLEAN.pdf
} 
TABLE 2 | Participant characteristics in DSBI feasibility study.

\begin{tabular}{lccc}
\hline & N (E4-) & N (E4+) & Total (N) \\
\hline ApoE & 6 & 6 & 12 \\
Gender: F & 6 & 4 & 10 \\
Gender: M & 1 & 1 & 2 \\
Age & $43.5(9.8)$ & $47.2(7.4)$ & $45.0(9.8)$ \\
Educ. years: & & & \\
0 & 0 & 1 & 1 \\
12 & 2 & 5 & 7 \\
18 & 4 & 0 & 4
\end{tabular}

There were six subjects who were ApoE4 positive and six who were ApoE4 negative. Average age was 45 (S.D. 9.8).

\section{Retinal $A \beta$}

The NeuroVision Retina $\mathrm{HD}$ is a fundus camera that is substantially equivalent to the FDA approved cameras currently utilized in clinical practice. In this procedure, a filter set matched to the fluorescence characteristics of curcumin is utilized for retinal amyloid plaque imaging in vivo. Quantitative analysis of $A \beta$ plaque number, area $\left(\mu \mathrm{m}^{2}\right)$ and distribution are performed from retinal images. For the acquisition, the same exposure settings and the same gain values are used for all images. The emission signals of $A \beta$ plaques stained with curcumin are compared to the background signals in the retinal tissue, to determine signal-to-background ratio.

At the visit, subjects had auto-fluorescence imaging and curcumin fluorescence imaging of the right retina. Patients were asked to take a standard over the counter oral vitamin $\mathrm{E}$ supplement for each retinal amyloid imaging visit, beginning at Day 1 and continuing through day 3 of imaging. Patients were dosed with curcumin twice daily for 2 days; At Day 1, patients commenced taking oral curcumin. On Day 2, subjects had another day of ingesting curcumin. On Day 3, subjects had auto-fluorescence imaging, and curcumin fluorescence imaging. NeuroVision calculated the retinal amyloid index in a blinded fashion for each subject.

\section{Statistical Analysis}

For cognitive, imaging analyses and fluid biomarker assessment, ApoE4 carriers and non-carriers were compared in terms of their age, educational level, clinical ratings, and neuropsychological test scores using Wilcoxon and Pearson's Chi-square tests. We also estimated Spearman rank correlations for each selected pairs of continuous measures. These correlation analyses were group by variable type: (1) cognitive vs. imaging; (2) cognitive vs. retinal and plasma; and (3) MRI vs. PET. We controlled the false discovery rate within each of these groups (Benjamini and Hochberg, 1995). This pilot study is not well powered. All analyses should be considered exploratory and any findings need to be confirmed with larger sample sizes. The sample size of $n=12$ provides approximately $80 \%$ power to detect only correlations larger than $\rho=0.84$ with two-sided FDR $\alpha=5 \%$ and assuming $90 \%$ of null hypotheses are true. Similarly, $n=6$ subjects per ApoE\&4 group provides approximately $80 \%$ power to detect only large standardized group differences of $\delta=2.66$.

\section{Results}

All 12 participants completed all required testing. Tables 2 and 3 show demographic characteristics for the 12 nondemented participants included in this study, grouped by APoE4. Of the 12 subjects, $50 \%(n=6)$ were ApoE4 carriers. All of the ApoE\&4 non-carriers were female, while four of the ApoE\&4 carriers were female. These two groups did not differ significantly in demographics, clinical ratings, or neuropsychological test scores. All subjects were

TABLE 3 | Cognitive and functional performance summaries by ApoE4 geneotype.

\begin{tabular}{|c|c|c|c|c|c|}
\hline & $\mathbf{N}$ & E4- & E4+ & Combined & $P$-value \\
\hline \multicolumn{6}{|l|}{ Direct testing } \\
\hline CAMCOG-DS & 12 & $58.2(23.4)$ & $56.5(20.3)$ & $57.3(20.9)$ & 0.81 \\
\hline RBANS Composite & 12 & $259.3(30.1)$ & $249.7(47.1)$ & $254.5(38.0)$ & 0.47 \\
\hline RBANS Digit span & 12 & $4.67(2.07)$ & $3.33(2.66)$ & $4.00(2.37)$ & 0.27 \\
\hline RBANS List recall & 12 & $14.67(3.98)$ & $11.83(3.54)$ & $13.25(3.89)$ & 0.18 \\
\hline Delayed Memory & 12 & $43.00(3.79)$ & $43.17(7.76)$ & $43.08(5.82)$ & 0.44 \\
\hline CANTAB-Total & 12 & $87.5(33.2)$ & $109.0(33.6)$ & $98.2(33.8)$ & 0.29 \\
\hline Dalton Dyspraxia & 12 & $205.7(41.3)$ & $177.7(49.9)$ & $191.7(46.1)$ & 0.31 \\
\hline \multicolumn{6}{|l|}{ Informant-based } \\
\hline ADAMS & 12 & $17.8(17.4)$ & $17.8(23.3)$ & 17.8 (19.6) & 0.81 \\
\hline Vineland-2 & 12 & 124.217 (32.9) & $96.767(24.5)$ & $110.4(31.2)$ & 0.24 \\
\hline
\end{tabular}

Higher score on all cognitive tests indicates better performance. The only exception is higher score on ADAMS, which indicates increased symptoms of anxiety, depression, agitation. ADAMS, Anxiety Depression and Mood Scale; CAMCOG-DS, Cambridge Examination for Mental Disorders of Older People with Down's Syndrome and Others with Intellectual Disabilities-Cognitive scale; Goodenough DAP, Goodenough-Harris Draw a Person test; RBANS, Repeatable Battery for the Assessment of Neuropsychological Status; (CANTAB), Cambridge Neuropsychological Test Automated Battery; OMQ-PF, Observer Memory Questionnaire (Parent Form). 
TABLE 4 | Amyloid PET and FDG PET with hippocampal volume and retinal amyloid index.

\begin{tabular}{|c|c|c|c|c|c|c|c|c|c|}
\hline Subject & Age & $\begin{array}{l}\text { Mental } \\
\text { age }\end{array}$ & ApoE 4 & $\begin{array}{l}\text { Amyloid PET } \\
\text { clinical read }\end{array}$ & $\begin{array}{c}\text { Grey matter } \\
\text { Amyloid PET } \\
\text { (SUVr) }\end{array}$ & $\begin{array}{l}\text { FDG PET } \\
\text { clinical read }\end{array}$ & $\begin{array}{c}\text { Average } \\
\text { Hippocampal } \\
\text { volume }\left(\mathrm{cm}^{3}\right)\end{array}$ & $\begin{array}{c}\text { Average } \\
\text { Hippocampal } \\
\text { Occupancy (\%) }\end{array}$ & $\begin{array}{c}\text { Retinal } \\
\text { amyloid index }\end{array}$ \\
\hline DP06 & 37 & 9 & E3-E3 & Negative & 0.938 & Normal & 3.52 & 73 & 1.63 \\
\hline DP01 & 32 & 7 & E3-E3 & Negative & 0.97 & Mildly hypo & 3.12 & 78 & 2 \\
\hline DP07 & 34 & 7 & E2-E4 & Negative & 0.988 & Normal & 3.37 & 75 & 2.47 \\
\hline DP08 & 39 & 5 & E3-E3 & Positive & 1.054 & Нypo & 3.19 & 82 & 1.8 \\
\hline DP02 & 45 & 3 & E2-E3 & Positive & 1.171 & Нypo & 2.99 & 45 & 2.2 \\
\hline DP12 & 45 & 6 & E3-E4 & Positive & 1.176 & Нyро & 2.91 & 75 & 1.83 \\
\hline DP05 & 48 & 8 & E3-E3 & Positive & 1.177 & Нypo & 3.47 & 77 & 1.68 \\
\hline DP11 & 47 & 7 & E3-E4 & Positive & 1.245 & Нypo & 3.48 & 71 & 2.34 \\
\hline DP13 & 50 & 8 & E3-E4 & Positive & 1.344 & Hypo & 3.14 & 60 & 1.58 \\
\hline DP04 & 55 & 6 & E3-E4 & Positive & 1.385 & Нypo & 3.01 & 45 & 1.7 \\
\hline DP03 & 52 & 7 & E3-E4 & Positive & 1.401 & Нypo & 3.25 & 51 & 2.2 \\
\hline DP09 & 60 & 7 & E3-E3 & Positive & 1.457 & Нyро & 2.73 & 60 & - \\
\hline
\end{tabular}

Areas with higher amyloid deposition have relative hypometabolism on FDG PET. The listing is sorted with respect to increasing grey matter amyloid PET signal.

amyloid positive, but to varying degrees. Table 4 provides a line listing of some of the key neuroimaging variables for each study participant sorted by posterior cingulate gyrus (PCG) amyloid PET. Figure $\mathbf{1}$ demonstrates how multimodal assessments are made across subjects in native space.

\section{Correlation Analyses}

Figure 2 shows the correlation between key neuroimaging, and age and cognition. Age was significantly associated with florbetapir (AV45) uptake in the gray matter $(r=0.963$, $p<0.001)$ and thalamus $(r=0.595, p<0.041)$, and HOC $(r=-0.662, p=0.019)$. Florbetapir uptake in the gray matter was also significantly correlated with OMQ PF $(r=-0.769$, $p=0.005032)$. FDG uptake in the thalamus was significantly correlated with CAMCOG, Digit Span, OMQ PF, RBANS, and Vineland (all $r>0.6$ and $p<0.01$ ). HOC was correlated with OMQ PF $(r=0.587, p=0.049)$. Only the correlations between FDG Thalamus and OMQ-PF; FDG Thalamus and Vineland; and Florbetapir (AV45) Gray Matter and age were significant at the 0.005 level $(r=0.776, r=0.776$, and $r=0.963$ respectively).

\section{Correlation Between Cognition, and Plasma $A \beta$ and Retinal Amyloid}

Figure 3 shows the correlation between key neuroimaging, and age and cognition. We found no significant correlations between plasma or retinal amyloid measures

\begin{tabular}{|l|l|l|}
\hline Subject & Caudate & Thalamus \\
DP03 & & \\
$\quad$ Amyloid Binding & 1.45 & 1.41 \\
$\quad$ FDG Metabolism & 1.17 & 1.22 \\
$\quad$ Volume & 3.06 & 8.16 \\
$\quad$ Percent Change & N/A & N/A \\
\hline DP04 & 1.39 & 1.26 \\
$\quad$ Amyloid Binding & 0.83 & 1.09 \\
$\quad$ FDG Metabolism & 4.42 & 9.34 \\
$\quad$ Volume & N/A & N/A \\
$\quad$ Percent Change & & \\
\hline DP05 & 1.53 & 1.33 \\
$\quad$ Amyloid Binding & 1.08 & 1.19 \\
$\quad$ FDG Metabolism & 3.67 & 7.43 \\
$\quad$ Volume & N/A & N/A \\
$\quad$ Percent Change & & \\
\hline DP06... & & \\
& &
\end{tabular}

FIGURE 1 | Left: multimodal comparisons can be made in native space within individual subjects longitudinally. Right: Amyloid PET, FDG PET, and volumetric MRI were successfully performed in adults with down syndrome (DS) to capture important structure-function relationships. 

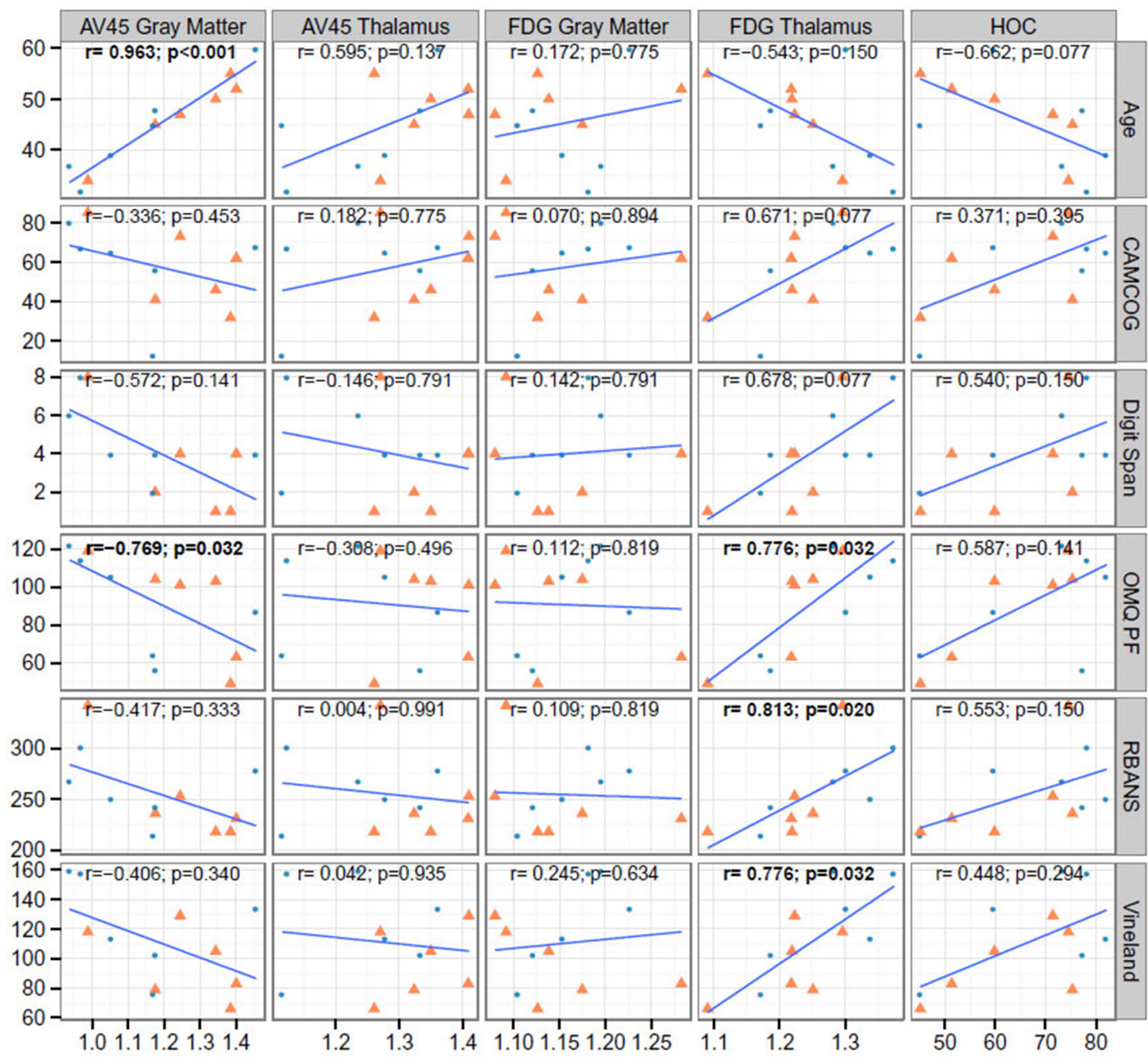

$$
\text { APOE } \cdot \cdot \text { e4- } \triangle \text { e4+ }
$$

FIGURE 2 | Correlations between cognitive and neuroimaging measures. The bold text indicates Spearman rank correlations $(r)$ that are significant at the 0.05 level after false discovery rate adjustment. AV45 $={ }^{18} \mathrm{~F}$-florbetapir.

and with age or cognition (Figure 3); nor between MRI and PET (Figure 4). We did find a significant correlation between A beta 42 and age $(r=0.602, p=$ $0.038)$.

\section{Correlation Between PET measures and HOC}

We found a significant negative correlation between Florbetapir (AV45) uptake in gray matter and HOC ( $r=-0.615, p=0.037)$. FDG uptake in thalamus and HOC were positively correlated $(r=0.671, p=0.020)$ see Figure 4.

\section{Retinal Amyloid Imaging}

We imaged amyloid plaques in the retina of all subjects in this small cohort, Figure 5. All subjects demonstrated amyloid positivity.

\section{Discussion}

Although limited in sample size, this small pilot study provides strong support for the feasibility of a multicenter longitudinal $\mathrm{AD}$ biomarker study in adults with DS. Our findings also show that prior to dementia onset, changes in volumetric MRI, 


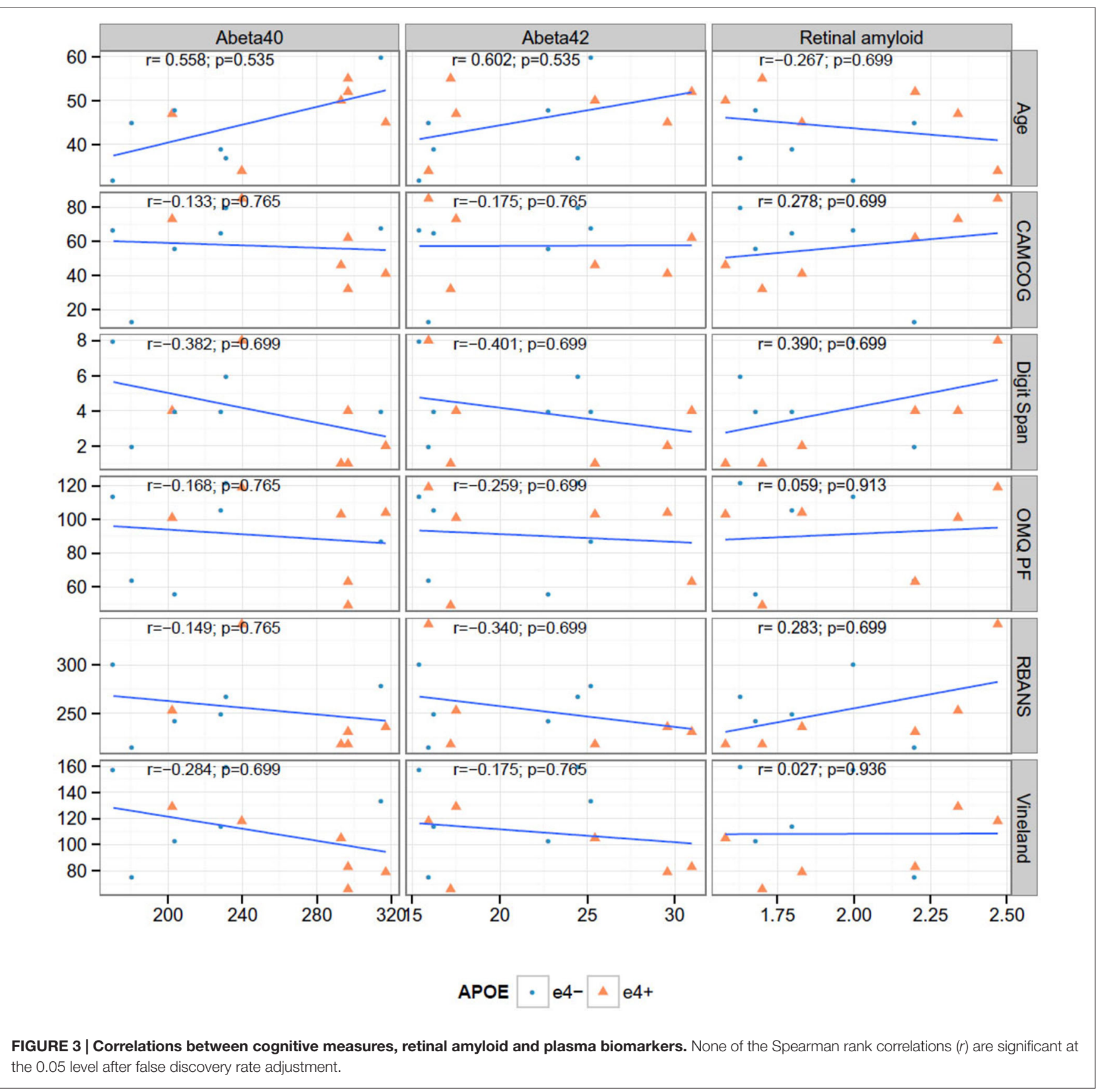

amyloid PET and FDG PET and plasma are detectable and consistent with preclinical AD in adults with DS. Adults with DS had elevated levels of plasma $A \beta 1-42$ concentrations and plasma $A \beta 1-42: A \beta 1-40$ ratios. These findings are consistent with previously published findings for individuals with DS (Schupf et al., 2010). Consistent with previous autopsy studies, most subjects demonstrated amyloid PET positivity reflecting fibrillar amyloid plaque deposition.

We also find adults with DS can tolerate amyloid- $\beta$ deposition without significant effects on cognitive functioning. This has been reported by others (Hartley et al., 2014) and likely reperesents the preclinical stage of $\mathrm{AD}$.

\section{Study Strengths}

We successfully studied AD biomarkers in all participants with DS, who, in the absence of an effective prevention treatment, are certain to develop symptoms of $\mathrm{AD}$. With this cohort, we confirm feasibility of a large-scale multicenter longitudinal study designed to characterize trajectories of cognitive decline. The fact that DS has native wild-type APP may make it more relevant to studying biomarkers applicable to the general sporadic AD. Additionally, we compared several different brain imaging and fluid biomarker measurements, as well as exploratory biomarkers such as retinal $A \beta$ imaging, to characterize some of the earliest biomarker changes associated with the predisposition to $\mathrm{AD}$. 



APOE $\cdot$ e4- $\triangle \mathrm{e} 4+$

FIGURE 4 | Correlations between PET and hippocampal occupancy (HOC). None of the Spearman rank correlations $(r)$ are significant at the 0.05 level after false discovery rate adjustment. AV45 $={ }^{18} \mathrm{~F}$-florbetapir.

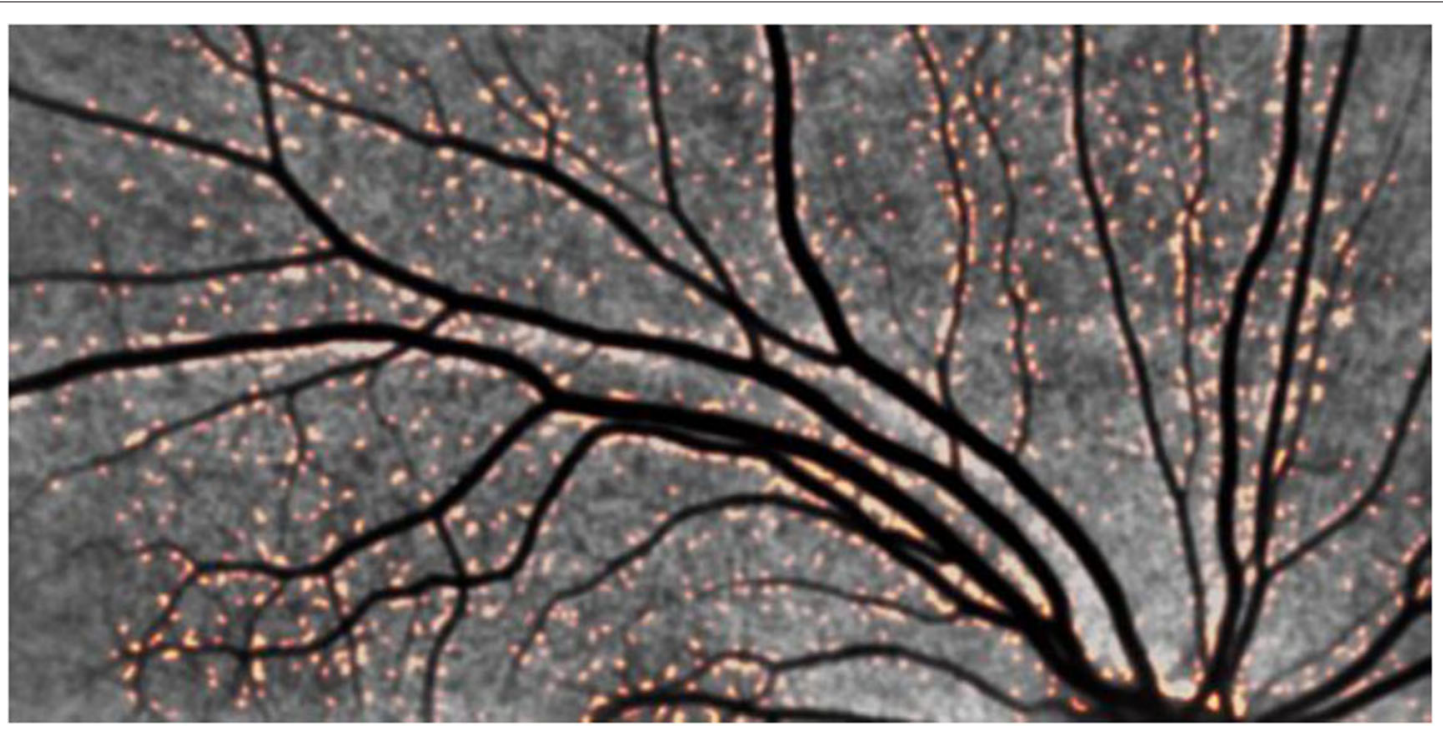

FIGURE 5 | Representative retinal images from an adult with DS demonstrating positive amyloid plaques in DS. Note the orange-colored puncta. The distribution in the vicinity of blood vessels is striking, pointing to a retinal manifestation of congophilic angiopathy.

\section{Limitations and Issues of Interpretation}

This study also has several limitations, including small sample size, absence of longitudinal data, and uncertainty in the extent to which our findings are generalizable to other causes of late-onset $\mathrm{AD}$. Although the retinal amyloid findings should be regarded as exploratory, the uncorrected significance levels, bilateral pattern, and resemblance to the pattern reported previously in patients with $\mathrm{AD}$ reduce the likelihood that they are attributable to the type I error associated with multiple regional comparisons. Although our findings are currently limited to DSBI pilot participants, we have sought to harmonize our biomarker measurements and undertake biological fluid assays in the same laboratory used by investigators in the study of other DS cohorts (LonDowns and Fundació Catalana de Síndrome de Down), thus providing complementary data and converging evidence in the preclinical study of AD in DS patients.
Additional studies are needed to clarify several issues: the extent to which the structural and functional abnormalities identified in young adults with $\mathrm{DS}$ at genetic risk for $\mathrm{AD}$ precede $\mathrm{A} \beta$ plaque deposition; whether these changes are neurodegenerative or developmental; whether or not there is any cerebral fibrillar $\mathrm{A} \beta$ deposition in young adults with DS.

\section{Conclusion}

Adults with DS have volumetric MRI, A $\beta$ PET, FDG PET and retinal $A \beta$ changes, along with plasma biomarker findings consistent with A $\beta 1-42$ overproduction. This study shows some of the earliest known AD biomarker changes in adults with DS and underscores the need for studies to clarify the earliest brain changes associated with the predisposition to AD. We have recently added Tau PET imaging to the set of biomarkers assessed in this cohort. Under the auspices of the DSBI pilot, we are continuing to characterize the 
age-related trajectory of biomarker changes associated with preclinical $\mathrm{AD}$ to set the stage for the first clinical trial of an anti-A $\beta$ therapy in the preclinical treatment of $\mathrm{AD}$ in adults with DS.

\section{Author Contributions}

MR, PSA, SN, RR and WM designed the study. MR, HW, JB, MD and RR executed the study,

\section{References}

Beacher, F., Daly, E., Simmons, A., Prasher, V., Morris, R., Robinson, C., et al. (2010). Brain anatomy and ageing in non-demented adults with down's syndrome: an in vivo MRI study. Psychol. Med. 40, 611-619. doi: 10. 1017/s0033291709990985

Benjamini, Y., and Hochberg, Y. (1995). Controlling the false discovery rate: a practical and powerful approach to multiple testing. J. R. Stat. Soc. Series B 57, 289-300.

Blessed, G., Black, S. E., Butler, T., and Kay, D. W. (1991). The diagnosis of dementia in the elderly. A comparison of CAMCOG (the cognitive section of CAMDEX), the AGECAT program, DSM-III, the Mini-Mental State Examination and some short rating scales. Br. J. Psychiatry 159, 193-198. doi: 10.1192/bjp.159.2.193

Brewer, J. B., Magda, S., Airriess, C., and Smith, M. E. (2009). Fully-automated quantification of regional brain volumes for improved detection of focal atrophy in Alzheimer disease. AJNR Am. J. Neuroradiol. 30, 578-580. doi: 10. 3174/ajnr.a1402

Coppus, A. M., Evenhuis, H. M., Verberne, G. J., Visser, F. E., Arias-Vasquez, A., Sayed-Tabatabaei, F. A., et al. (2008). The impact of apolipoprotein E on dementia in persons with down's syndrome. Neurobiol. Aging 29, 828-835. doi: 10.1016/j.neurobiolaging.2006.12.013

Dalton, A. J. (1992). Dementia in down syndrome: methods of evaluation. Prog. Clin. Biol. Res. 379, 51-76.

Deb, S., Braganza, J., Norton, N., Williams, H., Kehoe, P. G., Williams, J., et al. (2000). APOE epsilon 4 influences the manifestation of Alzheimer's disease in adults with down's syndrome. Br. J. Psychiatry 176, 468-472. doi: 10.1192/bjp. 176.5.468

Donohue, M. C., Moghadam, S. H., Roe, A. D., Sun, C. K., Edland, S. D., Thomas, R. G., et al. (2014). Longitudinal plasma amyloid beta in Alzheimer's disease clinical trials. Alzheimers. Dement. doi: 10.1016/j.jalz.2014.07.156 [Epub ahead of print].

Esbensen, A. J., Rojahn, J., Aman, M. G., and Ruedrich, S. (2003). Reliability and validity of an assessment instrument for anxiety, depression and mood among individuals with mental retardation. J. Autism Dev. Disord. 33, 617-629. doi: 10. 1023/b:jadd.0000005999.27178.55

Fischl, B., Salat, D. H., Busa, E., Albert, M., Dieterich, M., Haselgrove, C., et al. (2002). Whole brain segmentation: automated labeling of neuroanatomical structures in the human brain. Neuron 33, 341-355. doi: 10.1016/S08966273(02)00569-X

Gonzalez, L. M., Anderson, V. A., Wood, S. J., Mitchell, L. A., Heinrich, L., and Harvey, A. S. (2008). The observer memory questionnaireparent form: introducing a new measure of everyday memory for children. J. Int. Neuropsychol. Soc. 14, 337-342. doi: 10.1017/s1355617708 08020x

Goodenough, F. L., and Harris, D. (1950). Studies in the psychology of children's drawings. II. 1928-1949. Psychol. Bull. 47, 369-433. doi: 10.1037/h0058368

Handen, B. L., Cohen, A. D., Channamalappa, U., Bulova, P., Cannon, S. A., Cohen, W. I., et al. (2012). Imaging brain amyloid in nondemented young adults with down syndrome using pittsburgh compound B. Alzheimers. Dement. 8, 496-501. doi: 10.1016/j.jalz.2011.09.229

Hartley, S. L., Handen, B. L., Devenny, D. A., Hardison, R., Mihaila, I, Price, J. C., et al. (2014). Cognitive functioning in relation to brain amyloid- $\beta$ in healthy adults with Down syndrome. Brain 137(Pt. 9), 2556-2563. doi: 10.1093/brain/ awu173 performed the research, analyzed the data and wrote the manuscript.

\section{Acknowledgments}

We thank the staff of the ADCS Biomarker and Imaging cores for technical help during the trial. This work was sponsored by funding from Janssen Research and Development, LLC and NIH AG047484 and AG010483.

Heister, D., Brewer, J. B., Magda, S., Blennow, K., McEvoy, L. K., and Alzheimer's Disease Neuroimaging, I. (2011). Predicting MCI outcome with clinically available MRI and CSF biomarkers. Neurology 77, 1619-1628. doi: 10.1212/wnl. 0b013e3182343314

Hof, P. R., Bouras, C., Perl, D. P., Sparks, D. L., Mehta, N., and Morrison, J. H. (1995). Age-related distribution of neuropathologic changes in the cerebral cortex of patients with down's syndrome. Quantitative regional analysis and comparison with Alzheimer's disease. Arch. Neurol. 52, 379-391. doi: 10. 1001/archneur.1995.00540280065020

Hon, J., Huppert, F. A., Holland, A. J., and Watson, P. (1999). Neuropsychological assessment of older adults with down's syndrome: an epidemiological study using the Cambridge Cognitive Examination (CAMCOG). Br. J. Clin. Psychol. 38, 155-165. doi: 10.1348/014466599162719

Hyman, B. T., West, H. L., Rebeck, G. W., Lai, F., and Mann, D. M. (1995) Neuropathological changes in down's syndrome hippocampal formation. Effect of age and apolipoprotein E genotype. Arch. Neurol. 52, 373-378. doi: 10 1001/archneur.1995.00540280059019

Jovicich, J., Czanner, S., Greve, D., Haley, E., van der Kouwe, A., Gollub, R., et al. (2006). Reliability in multi-site structural MRI studies: effects of gradient nonlinearity correction on phantom and human data. Neuroimage 30, 436-443. doi: 10.1016/j.neuroimage.2005.09.046

Kovacevic, S., Rafii, M. S., Brewer, J. B., and Alzheimer's Disease Neuroimaging, I. (2009). High-throughput, fully automated volumetry for prediction of MMSE and CDR decline in mild cognitive impairment. Alzheimer Dis. Assoc. Disord. 23, 139-145. doi: 10.1097/wad.0b013e318192e745

Krasuski, J. S., Alexander, G. E., Horwitz, B., Rapoport, S. I., and Schapiro, M. B. (2002). Relation of medial temporal lobe volumes to age and memory function in nondemented adults with down's syndrome: implications for the prodromal phase of Alzheimer's disease. Am. J. Psychiatry 159, 74-81. doi: 10.1176/appi. ajp.159.1.74

Lemere, C. A., Blusztajn, J. K., Yamaguchi, H., Wisniewski, T., Saido, T. C., and Selkoe, D. J. (1996). Sequence of deposition of heterogeneous amyloid betapeptides and APO E in down syndrome: implications for initial events in amyloid plaque formation. Neurobiol. Dis. 3, 16-32. doi: 10.1006/nbdi.1996. 0003

Luciana, M. (2003). Practitioner review: computerized assessment of neuropsychological function in children: clinical and research applications of the Cambridge Neuropsychological Testing Automated Battery (CANTAB). J. Child. Psychol. Psychiatry 44, 649-663. doi: 10.1111/1469-7610.00152

Moran, J. A., Rafii, M. S., Keller, S. M., Singh, B. K., Janicki, M. P., American Academy of Developmental, M., et al. (2013). The national task group on intellectual disabilities and dementia practices consensus recommendations for the evaluation and management of dementia in adults with intellectual disabilities. Mayo Clin. Proc. 88, 831-840. doi: 10.1016/j.mayocp.2013.04.024

Mullins, D., Daly, E., Simmons, A., Beacher, F., Foy, C. M., Lovestone, S., et al. (2013). Dementia in down's syndrome: an MRI comparison with Alzheimer's disease in the general population. J. Neurodev. Disord. 5:19. doi: 10.1186/18661955-5-19

Ness, S., Rafii, M., Aisen, P., Krams, M., Silverman, W., and Manji, H. (2012). Down's syndrome and Alzheimer's disease: towards secondary prevention. Nat. Rev. Drug Discov. 11, 655-656. doi: 10.1038/nrd3822

Parker, S. E., Mai, C. T., Canfield, M. A., Rickard, R., Wang, Y., Meyer, R. E., et al. (2010). Updated national birth prevalence estimates for selected birth defects in the united states, 2004-2006. Birth Defects Res. A Clin. Mol. Teratol. 88, 1008-1016. doi: 10.1002/bdra.20735 
Pietrini, P., Dani, A., Furey, M. L., Alexander, G. E., Freo, U., Grady, C. L., et al. (1997). Low glucose metabolism during brain stimulation in older down's syndrome subjects at risk for Alzheimer's disease prior to dementia. Am. J. Psychiatry 154, 1063-1069. doi: 10.1176/ajp.154.8.1063

Prasher, V. P., Sajith, S. G., Rees, S. D., Patel, A., Tewari, S., Schupf, N., et al. (2008). Significant effect of APOE epsilon 4 genotype on the risk of dementia in Alzheimer's disease and mortality in persons with down syndrome. Int. J. Geriatr. Psychiatry 23, 1134-1140. doi: 10.1002/gps.2039

Randolph, C., Tierney, M. C., Mohr, E., and Chase, T. N. (1998). The Repeatable Battery for the Assessment of Neuropsychological Status (RBANS): preliminary clinical validity. J. Clin. Exp. Neuropsychol. 20, 310-319. doi: 10.1076/jcen.20.3. 310.823

Sabbagh, M. N., Chen, K., Rogers, J., Fleisher, A. S., Liebsack, C., Bandy, D., et al. (2015). Florbetapir PET, FDG PET and MRI in down syndrome individuals with and without Alzheimer's dementia. Alzheimers Dement. 11, 994-1004. doi: 10.1016/j.jalz.2015.01.006

Sabbagh, M. N., Fleisher, A., Chen, K., Rogers, J., Berk, C., Reiman, E., et al. (2011). Positron emission tomography and neuropathologic estimates of fibrillar amyloid-beta in a patient with down syndrome and Alzheimer disease. Arch. Neurol. 68, 1461-1466. doi: 10.1001/archneurol.2011.535

Schapiro, M. B., Azari, N. P., Grady, C. L., Haxby, J. V., and Horwitz, B. (1992b). Down syndrome: differentiating mental retardation and dementia with brain imaging techniques. Prog. Clin. Biol. Res. 379, 103-122.

Schapiro, M. B., Haxby, J. V., and Grady, C. L. (1992a). Nature of mental retardation and dementia in down syndrome: study with PET, CT and neuropsychology. Neurobiol. Aging 13, 723-734. doi: 10.1016/01974580(92)90096-g

Schupf, N., Kapell, D., Lee, J. H., Zigman, W., Canto, B., Tycko, B., et al. (1996). Onset of dementia is associated with apolipoprotein E epsilon4 in down's syndrome. Ann. Neurol. 40, 799-801. doi: 10.1002/ana.410400518

Schupf, N., Patel, B., Pang, D., Zigman, W. B., Silverman, W., Mehta, P. D., et al. (2007). Elevated plasma beta-amyloid peptide Abeta(42). levels, incident dementia and mortality in down syndrome. Arch. Neurol. 64, 1007-1013. doi: 10.1001/archneur.64.7.1007

Schupf, N., Patel, B., Silverman, W., Zigman, W. B., Zhong, N., Tycko, B., et al. (2001). Elevated plasma amyloid beta-peptide 1-42 and onset of dementia in adults with down syndrome. Neurosci. Lett. 301, 199-203. doi: 10.1016/s03043940(01)01657-3
Schupf, N., Zigman, W. B., Tang, M. X., Pang, D., Mayeux, R., Mehta, P., et al. (2010). Change in plasma Ass peptides and onset of dementia in adults with down syndrome. Neurology 75, 1639-1644. doi: 10.1212/wnl. 0b013e3181fb448b

Sheehan, R., Sinai, A., Bass, N., Blatchford, P., Bohnen, I., Bonell, S., et al. (2015). Dementia diagnostic criteria in down syndrome. Int. J. Geriatr. Psychiatry 30, 857-863. doi: 10.1002/gps.4228

Sled, J. G., Zijdenbos, A. P., and Evans, A. C. (1998). A nonparametric method for automatic correction of intensity nonuniformity in MRI data. IEEE Trans. Med. Imaging. 17, 87-97. doi: 10.1109/42.668698

Smith, P. J., Need, A. C., Cirulli, E. T., Chiba-Falek, O., and Attix, D. K. (2013). A comparison of the Cambridge Automated Neuropsychological Test Battery (CANTAB) with "traditional" neuropsychological testing instruments. J. Clin. Exp. Neuropsychol. 35, 319-328. doi: 10.1080/13803395.2013. 771618

Sparrow, S., and Havis, Y. (2005). Social care. Shared issues. Interview by stuart shepherd. Health Serv. J. 115, 32-33.

Sperling, R. A., Jack, C. R. Jr., and Aisen, P. S. (2011). Testing the right target and right drug at the right stage. Sci. Transl. Med. 3:111 cm133. doi: 10 . 1126/scitranslmed.3002609

Teipel, S. J., Alexander, G. E., Schapiro, M. B., Möller, H. J., Rapoport, S. I., and Hampel, H. (2004). Age-related cortical grey matter reductions in non-demented down's syndrome adults determined by MRI with voxel-based morphometry. Brain 127, 811-824. doi: 10.1093/brain/ awh101

Conflict of Interest Statement: The primary sponsor of the study, Janssen, provided input on study design as well as review of this manuscript. All authors had full access to all the data in the study and had final responsibility for the decision to submit for publication. Seth Ness is an employee of Janssen.

Copyright (c) 2015 Rafii, Wishnek, Brewer, Donohue, Ness, Mobley, Aisen and Rissman. This is an open-access article distributed under the terms of the Creative Commons Attribution License (CC BY). The use, distribution and reproduction in other forums is permitted, provided the original author(s) or licensor are credited and that the original publication in this journal is cited, in accordance with accepted academic practice. No use, distribution or reproduction is permitted which does not comply with these terms. 